\title{
Hiperplasia fibrosa inflamatoria y posibles factores asociados en adultos mayores
}

Rodríguez AF, Sacsaquispe SJ. Hiperplasia fibrosa inflamatoria y posibles factores asociados en adultos mayores. Rev Estomatol Herediana 2005; 15 (2) : 139 - 144.

\section{RESUMEN}

El propósito del estudio fue determinar la prevalencia de hiperplasia fibrosa inflamatoria (HFI) asociada al uso de prótesis dental removible y sus factores asociados como: sexo, grado de instrucción, edad, compromiso sistémico, estado nutricional e higiene oral del sujeto; tipo y tiempo de uso de la prótesis, tamaño y ubicación de la lesión en adultos mayores residentes del Albergue Central "Ignacia Rodulfo Vda. de Canevaro". Se evaluaron 91 sujetos mayores de 60 años $(80,67 \pm 7,65)$, que portaban prótesis dental removible y se registraron aquellos que presentaban HFI. Se midieron las lesiones, se registró la localización de éstas, se determinó la higiene oral mediante el índice de O'Leary y el índice de masa corporal. El 26,4\% presentaba HFI y la

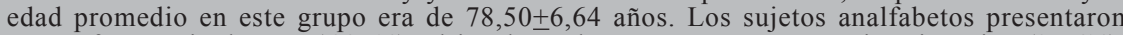
mayor frecuencia de HFI $(66,7 \%)$, al igual que los que tenían compromiso sistémico $(27,6 \%)$, mala higiene oral (34\%) y estado nutricional normal $(27,8 \%)(p>0,05)$. Los portadores de prótesis total inferior y parcial superior presentaron lesión en 33,3\% y $31,3 \%$ respectivamente. El promedio en tiempo de uso fue de $19,90 \pm 17,27$ años y del tamaño de cada lesión fue de $2,29 \mathrm{cms}$ en el sector ántero-superior (localización más frecuente: 58,6\%). En conclusión, el estudio demuestra que la HFI se desarrolló en sujetos de menor edad, con mala higiene oral, con compromiso sistémico y que no tiene relación con el tipo de prótesis y el tiempo de uso.

Palabras clave: HIPERPLASIA GINGIVAL. epidemiología / DENTADURA PARCIAL REMOVIBLE / DENTADURA COMPLETA.

Inflammatory fibrous hyperplasia and associated factors related to the use of dentures in the elderly

ABSTRACT

The purpose of the study is to determine the prevalence of fibrous inflammatory hyperplasia (IFH) in elder persons wearing dentures, and factors associated to gender, education, age, systemic compromise, nutrition and oral hygiene, time of use of the prosthesis, and size and location of the lesion; in elder patients living at the "Ignacia Rodulfo Vda. of Canevaro" Elderly Home. 91 denture-wearing subjects over 60 years of age $(80.67+7.65)$ were examined and all subjects with IFH were registered. Wound location and size were recorded as well as the body mass index of each subject. Oral hygiene was determined using the O'Loery Index from the total sample. $26.4 \%$ developed IFH and the average age was $78.50+6.64$ years. Illiterate subjects presented a higher frequency of IFH $(66.7 \%)$, similar to the systemically compromised subjects $(27.6 \%)$, subjects with poor oral hygiene $(34 \%)$ and normal nutritional state $(27.8 \%)(\mathrm{p}>0.05)$. Wounds were present in $33.3 \%$ of complete lower denture wearers and $31.3 \%$ of partial upper denture wearers. The average time of wear was $19.90+17.27$ years and the average wound size was $2.29 \mathrm{~cm}$. which was most frequently located $(58 . \overline{6} \%)$ in the anterior upper labial region. In conclusion, the study shows that the IFH developed in younger subjects, with poor oral hygiene and systemically compromised. No relationship between the type and time of use of the denture with IFH development was found.

Keywords: GINGIVAL HYPERPLASIA. epidemiology / REMOVABLE PARTIAL DENTURE COMPLETE DENTURE.

\section{Introducción}

El reemplazo de los dientes con aparatos protésicos no siempre está exento de riesgos, ya que un buen número de adultos mayores portadores de dentaduras artificiales presentan atrofia del proceso alveolar causada por desajuste protésico, lo que produce irritación mecánica de la mucosa que desencadena un proceso inflamatorio crónico que termina con la formación de tejido fibroso hiperplásico asintomático en las zonas de agresión, conocida como hiper-plasia fibrosa inflamatoria (HIF) o también llamada epulis fissuratum, épulis fisurado, por la presencia de surcos que corresponden a las zonas de compresión del borde protésico, las que deben ser eliminadas quirúrgicamente antes de que ocasionen la atrofia completa del hueso alveolar, ya que de ocurrir ello, significa- ría la imposibilidad de volver a sostener otra dentadura en esa arcada (1).

La HFI es una lesión que se desarrolla por la disminución del soporte (debido a la reabsorción alveolar) y la prótesis se profundiza poco a poco sobre el fondo de surco, ejerciendo una presión anormal sobre los tejidos blandos. Aquí se combinan dos lesiones: úlcera crónica e hiperplasia de tejido conjuntivo crónicamente inflamado. Su etiología está relacionada a la irritación crónica causada por los bordes de las prótesis. La mayoría de los casos han sido reportados entre la quinta y séptima década de vida, generalmente localizada en la parte anterior del maxilar superior (2-6). Clínicamente, el tejido hiperplásico suele formar lobulillos o pliegues y puede estar fisurado en el punto donde el reborde de la prótesis contacta con el tejido, en la base de las presiones lineales. Se localiza con mayor frecuencia en la zona anterior; son blandas, flácidas y móviles, y cuando existe ulceración, estas hiperplasias se muestran eritematosas (6).

Las prótesis removibles se sujetan a algunos dientes naturales mediante dispositivos no rígidos (retenedores), y a veces también descansan sobre el hueso cubierto de mucosa. Por estas razones, al morder sobre ellos se nota un pequeño rebote, y la masticación no es tan eficaz como con los dientes naturales. Con el tiempo, el hueso sobre el que éstos se apoyan cambia de forma, por lo que dichos aparatos se desadaptarán y podrían generar algunas molestias o ulceraciones que requieren un ajuste realizado por el odontólogo (7). Por otro lado, los adultos mayores son más pro- 
pensos a contraer este tipo de lesiones en la mucosa oral, entre ellas la HFI, debido a que muchos de ellos usan prótesis removibles. En la literatura se han reportado desórdenes de la mucosa oral en relación a la edad avanzada. Sin embargo, la edad, no es el único factor que se relaciona a la aparición de lesiones en la mucosa oral, pues otros hallazgos, como el trauma, medicamentos y falta de limpieza de las prótesis dentales removibles pueden desarrollar condiciones para la aparición de éstas $(2,8)$.

El propósito del estudio fue determinar la prevalencia de hiperplasia fibrosa inflamatoria asociada al uso de prótesis dental removible y sus factores asociados en adultos mayores.

\section{Materiales y método}

Se evaluaron adultos mayores (mayores de 60 años) portadores de prótesis dental removible del Albergue Central "Ignacia Rodulfo Vda. de Canevaro" que desearon participar en el estudio (firmaron Carta de Consentimiento Informado) y se excluyeron a sujetos que presentaran algún trastorno psiquiátrico.

En cada uno de los sujetos se estudiaron algunos factores relacionados con el HFI, como: edad, sexo, grado de instrucción, estado sistémico, estado nutricional e higiene oral del paciente; tipo y antigüedad de la prótesis, y en relación con la lesión su tamaño y ubicación anatómica en los rebordes alveolares de los maxilares.

Previa autorización del Comité de Ética y de la institución, se realizó un estudio piloto y calibración en la identificación de las lesiones orales. Luego se procedió al examen clínico de los participantes evaluando la extensión de las lesiones (longitud) en centímetros y para la ubicación de la lesión se registró considerando regiones anatómicas.

Procedimiento clínico:

Los exámenes bucales fueron realizados por un solo examinador y participaron en el estudio todos los sujetos que portaban prótesis dental removible para realizar las evaluaciones orales, las cuales fueron registradas mediante fotografías intraorales. Simultáneamente se registraron los datos clínicos en una ficha confeccionada especialmente para el estudio, como por ejemplo: su estado sistémico, estado nutricional, higiene oral de los participantes, y el tipo y tiempo de uso de la prótesis.
Para determinar el estado nutricional se obtuvo el índice de masa corporal y se registró peso/talla. Para evaluar su higiene oral se utilizó el índice de O'Leary et al. para medir la presencia de placa por medio de la observación visual o deslizando la sonda periodontal sobre las piezas dentarias y/o la prótesis del sujeto.

Análisis estadístico: El análisis estadístico de los datos se realizó de acuerdo a las variables estudiadas usando el programa SPSS v 11. Para determinar la prevalencia utilizando la estadística descriptiva y hallar la relación de las diferentes variables con la HFI, se utilizó la prueba de chi-cuadrado. Además se realizó una estimación en el programa E VIEWS v 5,1.

\section{Resultados}

Participaron en el estudio 91 adultos mayores entre 62 y 98 años de edad,

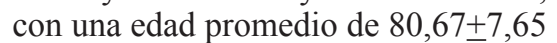
años, que usaban prótesis dental removible. Del total de sujetos examinados se observó que hubieron más mujeres $(62,6 \%)$ que varones $(37,4 \%)$, mientras que de acuerdo al grado de instrucción de cada paciente se observó que la mayoría de estos sujetos no eran analfabetos $(96,7 \%)$. Del total de sujetos en relación al tipo de prótesis dental removible se encontró lo siguiente: 65 de los 91 sujetos utilizan prótesis total superior, 40 utilizan prótesis total inferior, 24 utilizan prótesis parcial superior y 19 utilizan prótesis parcial inferior, con un tiempo promedio de uso en años de $12,78 \pm 12,62$.

De todos los sujetos examinados, 24 $(26,4 \%)$ presentaron al menos una lesión de HFI, mientras que $67(73,6 \%)$ no presentaron lesión. El número promedio de lesiones por adulto mayor con al menos una lesión fue de 1,21+0,51. De los sujetos que se encuentran entre los 70 a 79 años, el 37,5\% de éstos presentó HFI (Fig. 1). En la tabla 1 al comparar la presencia de HFI según sexo, se encontró una mayor frecuencia de lesiones en varones $(32,4 \%)$ y una menor frecuencia de lesiones en sujetos no analfabetos $(25 \%)$ y la edad en los adultos mayores con HFI fue menor $(78,50+6,64$ años) que en el grupo sin lesiones. Además se encontró una mayor frecuencia de lesiones en sujetos sistémicamente comprometidos $(27,6 \%)$ que en aquellos sin ninguna alteración sistémica $(24,2 \%)$; en aquellos con estado nutricio-nal normal $(27,8 \%)$ que en desnutridos $(24,3 \%)$, y en aquellos que presentaron mala higiene oral $(34,0 \%)$ en comparación con los que presentaron buena higiene oral $(12,0 \%)$.

Al comparar los portadores y no portadores se identificó que fue más frecuente la presencia de HFI en los no portadores de prótesis total superior (30,8\%) y el $33,3 \%$ de los portadores de prótesis total inferior ( $p>0,05)$. Tampoco se encontró diferencia entre los portadores y no portadores de prótesis total con HFI. Al evaluar el uso de prótesis parcial superior se encontró mayor número de lesiones $(31,3 \%)$ en los portadores que los que no usaban, y entre los portadores y no portadores de prótesis parcial inferior fue muy similar (26,4\% y $26,3 \%$ respectivamente). El tiempo promedio de uso de la prótesis en los sujetos que presentaban lesiones de HFI fue mayor (19,90+17,27 años) que en los que no las presentaban, aunque no hubo diferencia estadísticamen-te significativa entre ambos grupos (Tabla 2).

En la figura 2, la correlación de Spearman $(0,133)$ muestra que la mayoría de sujetos que utilizaron sus próte-

Tabla 1. Prevalencia de hiperplasia fibrosa inflamatoria en adultos mayores de acuerdo al sexo, edad y grado de instrucción.

\begin{tabular}{|c|c|c|c|c|}
\hline covariables & $\begin{array}{l}\text { presente } \\
\mathrm{n}(\%)\end{array}$ & $\begin{array}{l}\text { ausente } \\
\mathrm{n}(\%)\end{array}$ & $\begin{array}{c}\text { total } \\
\mathrm{n}\end{array}$ & valor $\mathrm{p}$ \\
\hline $\operatorname{sexo}^{*}$ & & & & 0,317 \\
\hline mujer & $13(22,8)$ & $44(77,2)$ & 57 & \\
\hline varon & $11(32,4)$ & $23(67,6)$ & 34 & \\
\hline grado de instrucción** & & & & 0,169 \\
\hline analfabeto & $2(66,7)$ & $1(33,3)$ & 3 & \\
\hline no anafabeto & $22(25,0)$ & $66(75,0)$ & 88 & \\
\hline edad (años cumplidos)*** & & & & 0,115 \\
\hline \multirow[t]{2}{*}{ media $+\mathrm{DE}$} & $78,50 \pm 6,64$ & $81,45 \pm 8,14$ & $80,67 \pm 7,85$ & \\
\hline & $\begin{array}{l}\text { ción estándar } \\
\text { chi-cuadrado }\end{array}$ & $\begin{array}{l}* * \text { prueba ex } \\
* * * \text { prueba } \mathrm{t}\end{array}$ & $\begin{array}{l}\text { a de Fisher } \\
\text { student }\end{array}$ & \\
\hline
\end{tabular}


Tabla 2. Prevalencia de hiperplasia fibrosa inflamatoria en adultos mayores de acuerdo al tipo y tiempo de uso de prótesis dental.

\begin{tabular}{|c|c|c|c|c|}
\hline covariables & $\begin{array}{c}\text { presente } \\
\text { n }(\%)\end{array}$ & $\begin{array}{c}\text { ausente } \\
\text { n }(\%)\end{array}$ & $\begin{array}{l}\text { total } \\
\mathrm{n}\end{array}$ & valor $\mathrm{p}$ \\
\hline uso de prótesis total superior* & & & & 0,189 \\
\hline si & $4(15,4)$ & $22(84,6)$ & 26 & \\
\hline no & $20(30,8)$ & $45(69,2)$ & 65 & \\
\hline uso de prótesis total inferior* & & & & 0,089 \\
\hline si & $17(33,3)$ & $34(66,7)$ & 51 & \\
\hline no & $7(17,5)$ & $33(82,5)$ & 40 & \\
\hline uso de prótesis parcial superior* & & & & 0,105 \\
\hline si & $21(31,3)$ & $46(68,7)$ & 67 & \\
\hline no & $3(12,5)$ & $21(87,5)$ & 24 & \\
\hline uso de prótesis parcial inferior* & & & & 0,995 \\
\hline si & $19(26,4)$ & $53(73,6)$ & 72 & \\
\hline no & $5(26,3)$ & $14(73,7)$ & 19 & \\
\hline uso de prótesis total bimaxilar* & & & & 0,052 \\
\hline si & $6(15,8)$ & $32(84,2)$ & 38 & \\
\hline no & $18(34,0)$ & $35(60,0)$ & 53 & \\
\hline tiempo de uso de la prótesis (años)** & & & & 0,131 \\
\hline media $+\mathrm{DE}$ & $19,90 \pm 17,27$ & $11,66 \pm 10,27$ & $13,48 \pm 12,69$ & \\
\hline
\end{tabular}

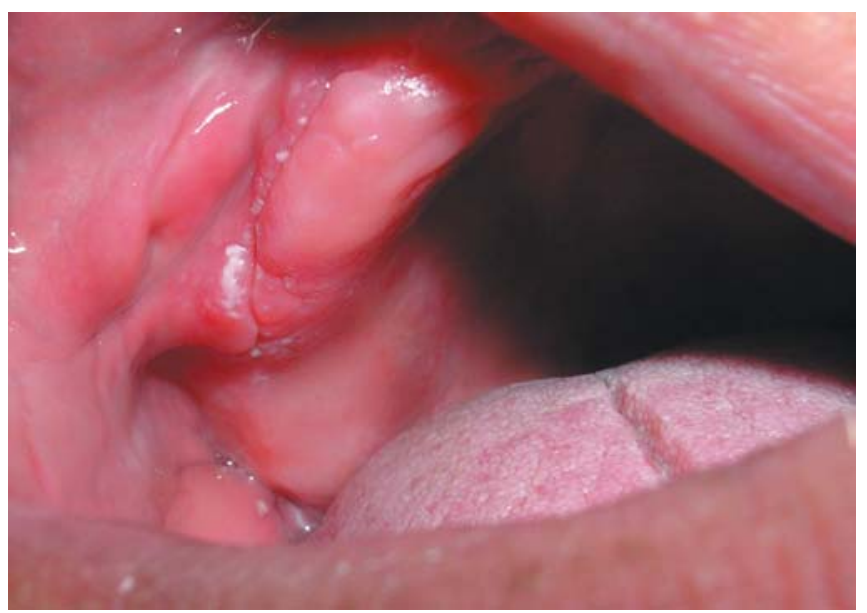

Fig 1. Hiperplasia fibrosa inflamatoria de reborde alveolar superior.

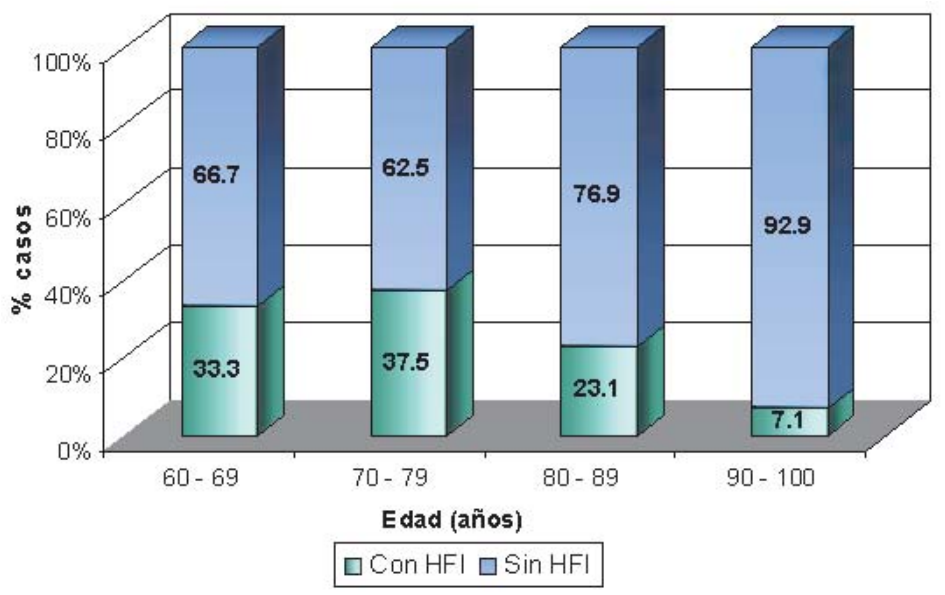

Fig. 2. Lesiones de hiperplasia fibrosa inflamatoria en adultos mayores.

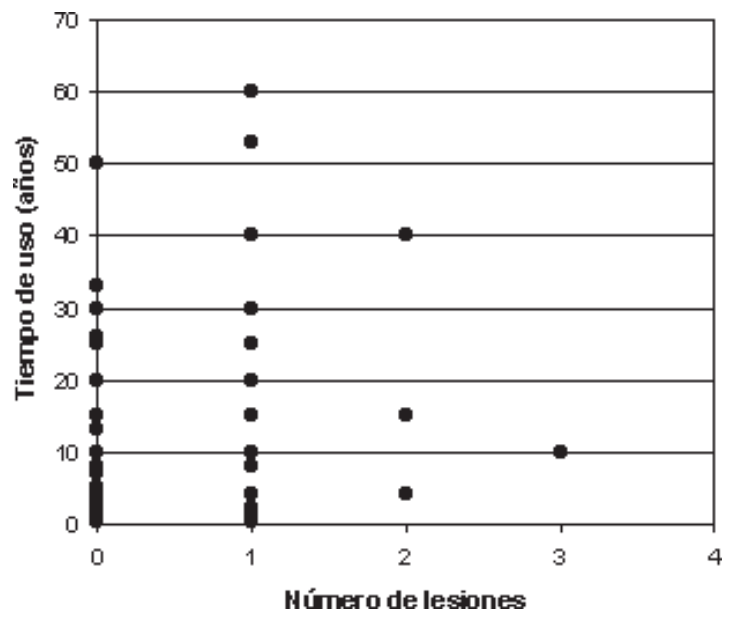

Fig. 3. Hiperplasia fibrosa inflamatoria de acuerdo al tiempo de uso de las prótesis dentales. *correlación de Spearman $=0,133 \quad \mathrm{p}=0,129 \quad \mathrm{n}=91$ 
sis desde los primeros meses hasta los 60 años de uso, habían desarrollado HFI. Sin embargo, también se encontró HFI en un sujeto con 3 lesiones, que había utilizado su prótesis por 10 años.

En relación a la distribución del número de lesiones de HFI según su ubicación se encontró una mayor frecuencia $(58,6 \%)$ en la aparición de esta lesión a nivel del fondo de surco vestibular en el sector antero superior. Finalmente, el tamaño promedio de las lesiones según su ubicación se encontró que el mayor promedio estuvo también en el sector antero superior con 2,29 $\pm 1,43$ centímetros.

Al hacer una estimación en el programa E VIEWS v 5.1; se encontró que el modelo a estimar es :

$h \mathrm{fi}=\beta_{1}{ }^{*}$ edad $+\beta_{2}{ }^{*}$ tdeuso $+\beta_{3}{ }^{*}$ higoral ${ }^{*}$ sexo $+\beta_{4}{ }^{*} \boldsymbol{p t}$

Y se encontró : Un incremento en la edad de un año reduce la probabilidad de desarrollar HFI en $1 \%$, un incremento en el tiempo de uso de 1 año, incre-menta la probabilidad de desarrollar HFI en 1\%, la condición de tener mala higiene oral y ser hombre incrementa la probabilidad de desarrollar HFI en $8 \%$ y el ser portador de una prótesis total incrementa la probabilidad de desarrollar HFI en 6\%.

\section{Discusión}

De los 91 sujetos examinados, se encontraron que el $26,4 \%$ presentó dicha lesión. En el estudio realizado por Meritxell (2) encontró 59 casos de épulis fisurado (39,8\%), mientras que en otros estudios el porcentaje es menor. Jainkittivong et al. (9) examinaron 500 sujetos adultos mayores, y encontraron que el $2 \%$ de éstos habían desarrollado hiperplasias inducidas por prótesis, Coehlo et al.(3) obtuvieron un 16,7\% de casos con HFI, Espinoza et al.(10) encontraron una prevalencia de $9,4 \%$ de HFI en 889 sujetos mayores de 65 años y García-Pola et al.(11) examinaron a 308 individuos, de los cuales se registraron 16 casos $(27,3 \%)$. Como se sabe la HFI es ocasionada debido a la utilización de prótesis dentales removibles mal ajustadas que se encuentran sobrextendidas debido a la reabsorción alveolar y que irritan crónicamente los tejidos de la mucosa del fondo de surco. La diefe- rencia encontrada con el estudio de Jainkittivong et al. (9) podria deberse a las condiciones de vida y salud que son diferentes a nuestra realidad, en cuanto a la educaión y acceso a los servicios de salud; mientras que en los otros estudios se encontraron resultados similares.

Del total de sujetos examinados, se observó que existe una frecuencia mayor de esta lesión en varones; sin embargo, no es estadísticamente significativa con respecto a su desarrollo en mujeres, aunque según la literatura revisada esta lesión es más frecuente en el sexo femenino. Stern (4) afirma que esta lesión es más frecuente en mujeres, y lo asocian al hecho de que las mujeres utilizan su prótesis por periodos más prolongados que los varones debido a no aceptar ser vistas sin ellas, probablemente esto juega un rol significante. Algunos autores han sugerido que posiblemente durante o después de la menopausia, existe una atrofia en el epitelio de la mucosa oral relacionado a la disminución en la secreción ovárica. El delgado epitelio oral atrófico ofrece poca protección contra varios irritantes, por lo tanto, es propenso a desarrollar una reacción inflamatoria como respuesta a una irritación crónica por una dentadura mal ajustada $(4,12)$. En un estudio realizado por García-Pola et al.(11), el desarrollo de la HFI se dio más en pacientes de sexo femenino, representando el 7\% de las 170 mujeres que fueron examinadas.

La frecuencia de HFI en los 91 sujetos, con respecto a su grado de instrucción fue de un $25 \%$ para los que no eran analfabetos. En cambio, se presentaron sólo tres sujetos analfabetos y dos de ellos presentaron HFI. Una de las variables que menciona un estudio realizado por Espinoza et al.(10) relacionado a la aparición de lesiones de la mucosa oral en adultos mayores, es la educación formal menor de 6 años, y se observó que el nivel educativo es independiente en la aparición de esta lesión, lo cual concuerda con nuestro estudio.

La edad promedio en la que prevaleció esta lesión fue de 78,5 años; como se sabe, la mayoría de estas lesiones se presentan en adultos mayores que utili- zan prótesis dental removible. En el presente estudio, al agrupar a los sujetos por decenios se muestra que sólo el $7,1 \%$ de los sujetos mayores de 90 años presentaron HFI. La edad promedio para el desarrollo de dicha lesión es a partir de los 72 años. Según Stern (4) afirma que la HFI puede presentarse en la quinta, sexta y sétima década de la vida; esto también lo afirman otros estudios similares a éste (12).

Dentro de las causas de alteraciones de la mucosa oral y estructuras adyacentes más comúnmente detectadas en el paciente geriátrico cabe mencionar las inherentes al proceso de envejecimiento, relativas al estado nutricional, secundarias a enfermedades sistémicas, medicación y relacionadas a procesos de adaptación o modificación de las estructuras bucales (1). Fue importante evaluar el compromiso sistémico de cada uno de los sujetos. Al comparar dicha condición con el desarrollo de la HFI, se encontró una mayor frecuencia de ésta en sujetos sistémicamente comprometidos $(27,6 \%)$. Dentro de las principales enfermedades que se encontraron fueron: hipertensión arterial, parkinson, diabetes, problemas cardíacos y otros (hipercolesterolemia, cefaleas, artritis, artrosis, entre otros), aunque todos estaban controlados. Si lo comparamos con los sujetos que no presentaron compromiso sistémico y sin embargo, desarrollaron HFI $(24,2 \%)$ ambos son muy similares; por lo que podemos concluir que el compromiso sistémico no es una condición determinante para el desarrollo de esta lesión, lo que fue demostrado en un estudio similar (10)

En cuanto al estado nutricional de cada sujeto también es una condicionante si el sujeto está o no sistémicamente comprometido. En nuestro estudio tanto los sujetos con malnutrición (37\%) y sin malnutrición (54\%) desarrollaron esta condición, similar a lo encontrado en otro estudio (10), por lo que se consideraría independiente para la aparición de la HFI.

La higiene oral también es un factor que influye en la aparición de esta lesión y de otras más. En nuestro estudio se encontró que de los que tuvieron 
mala higiene oral, el $34 \%$ presentó HFI, $\mathrm{y}$ en aquellos que presentaron buena higiene oral, sólo el $12 \%$ de éstos presentaron dicha lesión. Al respecto, Marchini et al. (13) realizaron un estudio en que relacionan las prácticas de higiene oral de las prótesis y la condición de los tejidos bucales, encontrándose una relación significativa entre la presencia de HFI, presencia de placa bacteriana y las prácticas de higiene de las prótesis, además del cuidado de la salud oral.

De todos los sujetos entrevistados, muchos de ellos no realizaban una limpieza adecuada de sus prótesis y estaban acostumbrados a dormir con ellas. Esto nos debe llevar a reflexionar como profesionales de la salud, que podemos prevenir la aparición de tales lesiones, si se le indicara al paciente de qué forma debe manipular e higienizar las prótesis así como el de acudir a sus controles para realizar ajustes en su oclusión.

El factor principal causante de la aparición y evolución de la HFI es el uso de prótesis dental removible, que se encuentran mal ajustada y que debido a la reabsorción alveolar ésta se intruye e irrita crónicamente la mucosa del fondo de surco. Esto lo afirman: Thomas (14), Meister et al. (15), Jha y Mathur (16), Keng y Loh (17), Krolls y McGinnis (18) y Cutright (19). En nuestro estudio, se encontró que no existe diferencia significativa en el tipo de prótesis para el desarrollo de la lesión. Sin embargo, los resultados muestran que los sujetos que utilizaban prótesis parcial removible, fueron los que más desarrollaron la HFI; esto podría explicarse debido a que muchas de estas prótesis fueron de acrílico sin armazón metálico inadecuadamente confeccionadas y en mal estado de conservación. También se encontró adultos mayores que utilizando prótesis dental removible no desarrollaron HFI, como: el 84,6\% de sujetos que utilizaban prótesis total superior y el $84,2 \%$ que usaban prótesis total bimaxilar. El sólo hecho de que un sujeto sea portador de prótesis removible, incrementa el riesgo para desarrollar HFI, además de otras patologías bucales como: queilitis angular, estomatitis sub- protésica, úlceras traumáticas, candidiasis oral, etc. $(2-4,10,12-19)$

Con respecto al factor anterior, es importante resaltar que el tiempo de uso en años de las prótesis removibles es un factor que predispone el desarrollo de varias lesiones orales. Muchas de las prótesis examinadas presentaban fracturas y deterioro del material por la antigüedad de éstas. A pesar de que en nuestros resultados, esta diferencia no fue estadísticamente significativa, cabe mencionar que el tiempo promedio de uso en años para los sujetos que sí presentaron la lesión fue de 19,90 años, esto implicaría que a menos años de antigüedad de la prótesis, es menor la probabilidad de desarrollar esta lesión. Este resultado es similar al encontrado en un estudio realizado por Coelho et al. (12) atribuyendo una mayor frecuencia de HFI a mayor tiempo de uso de la dentadura.

En nuestro estudio se observó que la HFI tuvo una predilección para desarrollarse en el fondo de surco del sector ántero-superior. Muchos estudios coinciden que la localización más frecuente es en la región del fondo de surco vestibular mandibular o maxilar. Keng y Loh (17) realizaron un estudio en donde la localización más frecuente era en la región ántero-inferior del fondo de surco vestibular, y lo asocia a los movimientos de deslizamiento horizontal de la dentadura debido a la masticación y deglución que generaría la irritación en esa zona, debido al borde anterior de la prótesis $(70 \%)$.

El tamaño de la lesión es variable. Algunas de las lesiones son pequeñas, pero pueden extenderse e involucrar gran parte de la longitud del vestíbulo (4). El tamaño promedio encontrado en nuestro estudio por localización fue de 2,29 $\mathrm{cms}$ en la región ántero superior vestibular (16). Este resultado es muy similar al encontrado en un estudio realizado por da Motta (20), donde el tamaño promedio de la lesión fue de $2,8 \mathrm{cms}$.

Debemos tomar en cuenta que los adultos mayores de por sí presentan cambios degenerativos con respecto a la mucosa oral. Esto es debido a que la salud oral de las personas de la tercera edad está influenciada por el tipo de die- ta que ingieren, y por ende tiene una repercusión en su estado general. Se han realizado investigaciones en aquellos sujetos desdentados, y son lo que ingieren dietas con bajo aporte de nutrientes, por tanto esto puede conllevar a una malnutrición en comparación de los que son dentados. Esto acarrea otro tipo de problemas de carácter estético y dificultad para masticar. En nuestro estudio se puede observar que el estado nutricional de los sujetos no es factor condicionante para el desarrollo de esta lesión.

La higiene oral sí tiene gran influencia en la aparición de esta y otras lesiones, entre las que se pudo observar: candidiasis oral, hiperplasia papilar inflamatoria, úlceras traumáticas, xerostomía o sensación de boca seca, lengua fisurada, queilitis angular. Generalmente dichas lesiones se ocasionan en aquellos donde las prótesis han sido confeccionadas sin los principios básicos de la oclusión.

Se debe tener en cuenta los factores de oclusión de cada sujeto, pues las personas que utilizan prótesis dental total suelen suponer que no requieren visitar al dentista. Esto es falso porque el proceso de reabsorción del hueso alveolar continúa a lo largo de los años, y algunos padecimientos suelen afectar este proceso, especialmente en la mandíbula, lo cual puede provocar problemas en la estabilidad de las dentaduras. Esto se debe muchas veces, a que los mismos odontólogos, no indican controles después de haber instalado las prótesis. Otro aspecto que se observó, fue que algunos sujetos fueron atendidos por técnicos dentales quienes sólo confeccionan prótesis, y en consecuencia no indicaron controles.

Es importante considerar que las enfermedades bucales que se detectan en adultos mayores no pasen por alto en una evaluación general de salud de éstos, sólo así podemos identificar y tratar adecuadamente al paciente. El odontólogo debe estar lo suficientemente capacitado para evaluar y tratar de rehabilitar al paciente bajo las normas y condiciones que deben cumplir las prótesis como: soporte, retención y estabi- 
lidad y restablecer la funciones : masticatoria, fonética y estética; de ser así, estaríamos previniendo el desarrollo de este tipo de lesiones.

\section{Agradecimiento}

A Donita R. Rodriguez Zegarra y Erick W. Lahura Serrano por su apoyo y colaboración en el análisis estadístico.

\section{Referencias bibliográficas}

1. Irigoyen E. Mosqueda A. Estomatología Geriátrica. 2005. Disponible en: http://www.drscope.com/pac/ mg-2/7/mg2t7_p41.htm

2. Tamarit-Borras M, Delgado-Molina E, Berini-Aytes L, Gay-Escoda C. Removal of hyperplastic lesions of the oral cavity. A retrospective study of 128 cases. Med Oral Patol Oral Cir Bucal 2005;10(2):151-62.

3. Coelho C, Sousa T, Dare A, Cardoso. Avaliação Preliminar das Lesões da Mucosa Bucal Associadas Ao Uso de Prótese Removible. Rev Odont Univ Ribeirão Preto, 1999; 3:1.

4. Stern D. Epulis Fissurattum. Setiembre 2005. Disponible en: http:/ /www.emedicine.com/derm/ topic654.htm

5. Buchner A. Begleiter A. The Predominance of Epulis Fissuratum in females. Quintessence International 1984; 7, 1-4

6. Sciubba J. Denture Estomatitis. Junio 2005. Disponible en: http:// www.emedicine.com/derm/ topic642.htm

7. Asamblea General del Ilustre Con- sejo General de Colegios de Odontólogos y Estomatólogos. Modelos guía de instrucciones de manejo, cuidado y mantenimiento de las prótesis dentales convencionales. Instrucciones de manejo, cuidado y mantenimiento de prótesis removibles. España 2000.

8. Sosa L, De Ondiz M, Maldonado C. Análisis retrospectivo sobre las patologías bucales que se evidenciaron en la comunidad que acudió al servicio de odontología, Hospital Victorino Santaella Ruiz. Los Teques. Estado Miranda, Venezuela. Periodo comprendido desde el 4to trimestre de 2000 al 3 er trimestre de 2004. Disponible en: http:// www.odontologia-online.com/ casos/part/LST/LST11/lst11.html

9. Jainkittivong A, AneksuK V, Langlais RP. Oral mucosal conditions in elderly dental patients. Oral Diseases 2002; 8(4):218-223

10. Espinoza I, Rojas R, Aranda W, Gamonal J. Prevalence of oral mucosal lesions in elderly people in Santiago, Chile. J Oral Pathol Med 2003; 32(10): 571-575

11. Garcia-Pola Vallejo MJ, Martinez DiazCanel AI, Garcia Martin JM, Gonzalez Garcia M. Risk factors for oral soft tissue lesions in an adult Spanish population. Community Dent Oral Epidemiol 2002;30(4):277-85.

12. Coelho CM, Sousa YT, Dare AM. Denture-related oral mucosal lesions in a Brazilian school of dentistry. J
Oral Reh 2004; 31(2):135-139.

13. Marchini L, Tamashiro E, Nascimento DF, Cunha VP. Self-reported denture hygiene of a sample of edentulous attendees at a University dental clinic and the relationship to the condition of the oral tissues. Gerodontology 2004; 21(4):226-8.

14. Thomas GA. Denture-induced fibrous inflammatory hyperplasia (epulis fissuratum): research aspects. Aust Prosthodont J 1993; 7:49-53.

15. Meister F Jr, Hickman TR, Davies EE. Surgical treatment of epulis fissuratum utilizing free gingival grafts: report of two cases. J Wis Dent Assoc 1977; 53(6):257-9.

16. Jha $\mathrm{T}$, Mathur RM. Inflammatory fibrous hyperplasia - a short case report. J Pierre Fauchard Acad 1990; 4(1):155.

17. Keng SB, Loh HS. The treatment of epulis fissuratum of the oral cavity by CO 2 laser surgery. J Clin Laser Med Surg 1992; 10(4):303-6.

18. Krolls SO, McGinnis JP Jr. Case presentation. Denture-induced fibrous hyperplasia (epulis fissuratum). Miss Dent Assoc J 1993; 49(3):18-9.

19. Cutright DE. The histopathologic findings in 583 cases of epulis fissuratum. Oral Surg Oral Med Oral Pathol 1974; 37(3):401-11.

20. da Motta RG, da Motta ZG. Epulis fissuratum. Conducta clinicacirúrgica. Rev Bras Odontol 1979; 36(2):44-7. 